\title{
Analysis of Dynamic Brain Networks Using VAR Models
}

\author{
Christian Moewes, Rudolf Kruse, and Bernhard A. Sabel
}

\begin{abstract}
In neuroscience it became popular to represent neuroimaging data from the human brain as networks. The edges of these (weighted) graphs represent a spatio-temporal similarity between paired data channels. The temporal series of graphs is commonly averaged to a weighted graph of which edge weights are eventually thresholded. Graph measures are then applied to this network to correlate them, e.g. with clinical variables. This approach has some major drawbacks we will discuss in this paper. We identify three limitations of static graphs: selecting a similarity measure, averaging over time, choosing an (arbitrary) threshold value. The latter two procedures should not be performed due to the loss of brain activity dynamics. We propose to work on series of weighted graphs to obtain time series of graph measures. We use vector autoregressive (VAR) models to facilitate a statistical analysis of the resulting time series. Machine learning techniques are used to find dependencies between VAR parameters and clinical variables. We conclude with a discussion and possible ideas for future work.
\end{abstract}

Keywords: Dynamic networks, elctroencephalography, neuroimaging, regression, vector autoregressive model.

\section{Introduction}

In the last decade, a new trend in neuroscience emerged which focuses on the analysis of complex functional brain networks (see e.g. [18]). These networks

Christian Moewes · Rudolf Kruse

Faculty of Computer Science, University of Magdeburg,

39114 Magdeburg, Germany

e-mail: cmoewes@ovgu.de, kruse@iws.cs.uni-magdeburg.de

Bernhard A. Sabel

Medical Faculty, University of Magdeburg,

39120 Magdeburg, Germany

e-mail: bernhard.sabel@med.ovgu.de

R. Kruse et al. (Eds.): Synergies of Soft Computing and Statistics, AISC 190, pp. 525-532 springerlink.com

(C) Springer-Verlag Berlin Heidelberg 2013 
are obtained from neuroimaging data by several technologies, e.g. electroencephalography (EEG), electrocorticography (ECoG), magnetoencephalography (MEG) or functional magnetic resonance imaging (fMRI). These methods record activities of brain regions (e.g. on the skull, on the brain meninges or inside of the brain). We denote these brain regions as variables. The sampling rates of the data highly depend on the chosen method ( $\mathrm{kHz}$ for EEG, MEG and $\mathrm{ECoG}, \mathrm{Hz}$ for fMRI).

Whenever two brain regions are co-active, they are connected to each other. This connection induces a complex brain network that gives some high-level representation of the really connected nervous cells of the brain. There is general consensus that the analysis of such brain networks will help to better understand the functionality of different brain centers and the brain as a whole [15. Clearly, if the dynamic behavior of such networks is ignored, then valuable information will be lost pertaining to the brain networks being studied.

While it is challenging to even define nodes for brain networks 2 , here this definition is explicitly given by a specific application. We discuss the analysis of dynamic brain networks obtained from patients' EEG. The patients from which we recorded EEGs have visual field deficits (visual impairments) that resulted from optic nerve damages 21. Our goal is to find network features that correlate with clinically relevant variables. Since we deal with (partly) blind subjects, we assume that it has an effect on their functional connectivity networks. We also hypothesis that there is a correspondence between the extend of the vision loss and the dynamics of the brain connectivity.

It is well-known that brain damage leads to significant and long-lasting neurological deficits, e.g. paralysis or blindness. Therefore, it is of interest to study the relationship between the structural network damage and the functional one that might be observable by neuroimaging methods. The first study related to this problem was performed by C. Stam in 2007 [16. His group analyzed the differences in EEG data between 15 patients with Alzheimer's disease (AD) and 13 control subjects. Functional connectivity was computed using synchronization likelihood (SL) 17 and the obtained brain network were measured by small-world network criteria 19. Correlating these measures with clinical variables, they showed that AD is characterized by a loss of small-world network characteristics. Note that they used an averaged network with thresholded edge weights to eventually obtain an unweighted graph.

While we also describe brain networks using SL (to facilitate the choice of one similarity measure between two EEG channels), a previous analysis with averaged unweighted networks did not result in high correlations using a variety of graph measures [7. So, in this paper we are going to extend this approach manifold: (1) we consider the complete series of EEG networks without averaging over time, (2) we study weighted networks without any edge weight threshold, (3) we use machine learning techniques to find dependencies between network measures and output variables instead of correlation coefficient analyses. 


\section{Functional Connectivity}

To obtain a complex brain network from neuroimaging data, it is necessary to define and measure functional connectivity of two brain regions. Estimating functional connectivity does not find causal connections inside the human brain. Functional connectivity can only be interpreted as statistical relationship between brain regions without implying any causal coherence 11. Naturally a variety of different functional connectivity methods can been found in the literature (see [20] for a good overview on EEG measures).

Here, we only introduce the concept of SL [17. Consider a multivariate time series (e.g. a multichannel EEG recording) of length $N$ with $n$ variables. Let measurement $x_{i, k}$ be observed at timestamp $i$ in channel $k$. Firstly, a time-delay embedding is computed by

$$
X_{i, k}=\left(x_{i, k}, x_{i+L, k}, x_{i+2 \cdot L, k}, \ldots, x_{i+(m-1) \cdot L, k}\right)
$$

where $L$ is the lag and $m$ the dimension of the embedding. These state vectors $X_{i, k}$ shall capture the relevant patterns of the signal. Now consider only two channels $A, B$. Then, the probability that $X_{i, k}$ are closer to each other than $\varepsilon$ is

$$
P_{i, k}^{\varepsilon}=\frac{1}{2\left(W_{2}-W_{1}\right)} \sum_{\substack{j \\ W_{1}<|i-j|<W_{2}}}^{N} \theta\left(\varepsilon-d\left(X_{i, k}, X_{j, k}\right)\right)
$$

where $d$ is typically the Euclidean distance. For each $k$ and $i$ the critical distance $\varepsilon_{i, k}$ is computed such that $P_{i, k}^{\varepsilon_{i, k}}=p_{\text {ref }}$ whereas $p_{\text {ref }} \ll 1$ is some user-defined threshold. Then, for each pair of points in time $(i, j)$ within $W_{1}<|i-j|<W_{2}$, the number of channels $H_{i, j}$ for which $d\left(X_{i, k}, X_{j, k}\right)<\varepsilon_{i, k}$ is computed by

$$
H_{i, j}=\theta\left(\varepsilon_{i, A}-d\left(X_{i, A}, X_{j, A}\right)\right)+\theta\left(\varepsilon_{i, B}-d\left(X_{i, B}, X_{j, B}\right)\right)
$$

where $\theta(x)=0$ if $x \leq 0$ and $\theta(x)=1$ for $x>0$. The synchronization likelihood is then given by

$$
S L_{i}=\frac{1}{2 p_{\text {ref }}\left(W_{2}-W 1\right)} \sum_{\substack{j \\ W_{1}<|i-j|<W_{2}}}^{N}\left(H_{i, j}-1\right)
$$

The set of free parameters for SL can be reduced down to a size of two by prior information about the frequency range and temporal resolution of the signal [10]. 


\section{Brain Graphs}

A brain graph is created when computing functional connectivity, e.g. using SL, for each pair of variables at a given point in time. Such a network simply serves as graphical representation of pairwise statistical dependencies among all variables. Typically, these networks are described by graph measures (e.g. density, clustering coefficient, average path length). Usually, the hope is that they might correlate to clinical variables. Due to simplicity, we demand that brain graphs are simple, i.e. they do not have any loops or multiple edges. Since dealing with SL, we know that the brain graphs are symmetric.

\subsection{The Meaning of Edges}

An edge represents some kind of statistical dependency between two brain regions, i.e. functional connectivity. The edge weight corresponds to the strength of the functional connectivity. Most measures are normalized to $[0,1]$ or $[-1,1]$ which enables a straightforward interpretation of an edge weight. Commonly, researchers do not use weighted edges for graph analysis. Instead an arbitrarily chosen threshold is used to cancel out edge with "low" weights. Clearly, the remaining edges are unweighted. Despite the loss of information, some researchers argue that one can show different effects with a binary graph [13].

\section{Critical Remark and Proposal}

So far, we just reported standard techniques that convert neuroimaging data into brain networks. Let us now consider a critique we face when dealing with this approach and how to handle this problem.

We mainly argue that averaging brain graphs over time is generally not beneficial. Averaging should only be permitted if the variations of the binary time series (unweighted edge) or the numerical time series (weighted edge) were close to zero and stationary. To illustrate this, just consider a graph with two nodes, one edge and a linear trend in the evolution of the edge weight. Then, averaging would diminish this important information. In our application, a static analysis did not show very high correlations [7. We will present much stronger correlations in Section 5 using the following approach.

Remember that the patients are at rest. Thus every EEG time series can have a different length and so does the series of networks. Now, recall that certain graph measures are applied to each network to find global relations between them and clinical variables. The series of networks is eventually transformed into a multivariate time series of real-valued graph measures. 
Then, the question arises how these new time series with different lengths can be correlated to the clinical variables. We propose to use a model-based representation of these time series as it is independent from its length.

EEG data in rest do not show any trend and so do the graph measures. Thus the easiest way to fit the time series of graph measures is to use vector autoregressive (VAR) models [8. A simple VAR model with $p$ lags is given by

$$
\mathbf{x}_{t}=c+\sum_{i=1}^{p} A_{i} \mathbf{x}_{t-i}+\epsilon_{t} .
$$

where $c$ is a constant, $A_{i}$ is a matrix storing the interdependence between every pair of variable at point $t-i$ and $\varepsilon_{t}$ is white noise. Its coefficients $A_{i}$ can be simply flattened as vector which can eventually be correlated to the output variables. In the next section we will evaluate this approach using a real-world application.

\section{Application and Experiments}

In our experiments we used EEG data from 25 visually impaired subjects suffering from optic nerve damages 21. Enabling the relation of EEG graph measures to clinical variables, so-called "visual field charts" were obtained from every patient. They indicate the location and size of the optic nerve damage 14]. Based on them, an expert defined the following clinical variables:

- proportion of intact/white sectors,

- proportion of relatively defected/gray sectors,

- proportion of absolutely defected/black sectors,

All of these were transformed by the cortical magnification function (CMF) [4] resulting into 3 further variables.

To preprocess the data we did the following steps in EEGLAB [5]:

- manually removal of noisy time frames at beginning/end of each recording,

- removal of uncommon EEG channels across all subjects (28 were used),

- high-pass filtering with cutoff frequency at $1 \mathrm{~Hz}$ to remove slow movements,

- notch filtering $50 \mathrm{~Hz}$ to cope with European power line frequency,

- low-pass filtering with cutoff frequency at $95 \mathrm{~Hz}$,

- re-referencing by the average electrode,

- down-sampling to $150 \mathrm{~Hz}$ to reduce the costs of SL computation,

- removal of biological artifacts using independent component analysis 9.

Biological artifacts that stem from electromyographic (EMG) or electrocardiograph (EKG) signal appear as noise in the recorded EEG signal in all variations. For EMG/ECG removal, ICA was applied to very carefully remove noisy components. 
We used FIR filters to obtain the conventional separation into frequency bands. They are typically associated with different brain states [6]. These bands are $\delta: f \in(1,4] \mathrm{Hz}, \theta: f \in(4,7] \mathrm{Hz}, \alpha: f \in[8,12] \mathrm{Hz}, \beta: f \in[13,30] \mathrm{Hz}$, $\gamma: f \in[30,50] \mathrm{Hz}$ and $\mu: f \in[8,13] \mathrm{Hz}$. We expected the clinical variables to be somehow explainable by functional connectivity changes in these bands. Functional connectivity was established by SL [17] for each frequency band. We used an outer window length of $W_{2}=3 \mathrm{~s}$ and a reference probability of $p_{\text {ref }}=0.02$. To capture most of the dynamics, the sliding window shifted every $.5 \mathrm{~s}$, i.e. an overlay of $5 / 6$. Note that a statistical analysis of the averaged graphs has been published by these authors [7.
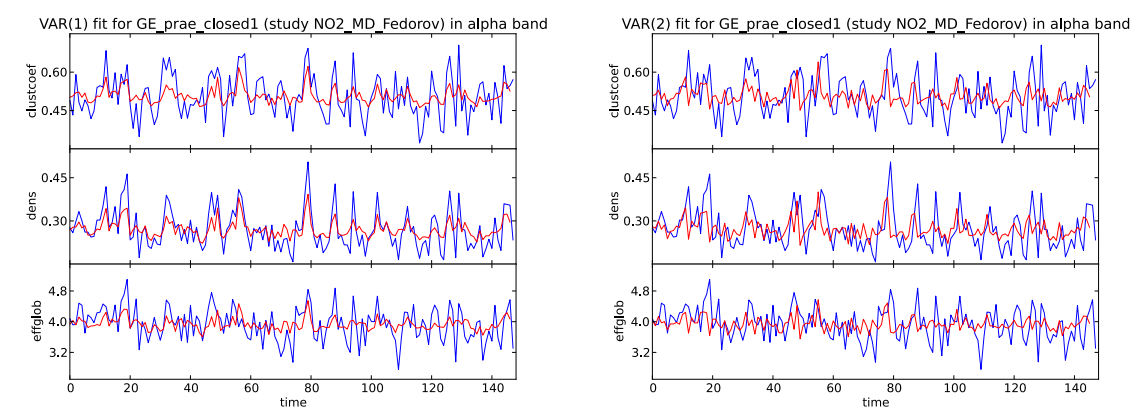

Fig. 1 Original and fitted time series of graph measures in the $\alpha$ band of one subject for $p=1,2$ on the left and right side, respectively

We applied 3 measures to every brain graph 3, i.e. average clustering coefficient, density and global efficiency, resulting in a multivariate time series for each subject and each frequency band. Every time series was modeled by a VAR model with $p=1,2$ for simplicity. Thus we obtained $p \cdot n \cdot n=9$ and 18 parameters, respectively, describing the dynamics of the corresponding multivariate time series. Figure 1 shows an example of these time series. Every clinical variable served as variable being depended from these inputs. We used ridge regression (a penalized version of least-squares) with generalized cross validation on different penalizer $\alpha \in\{.1, .2, \ldots, .9,1\}[12$. Thus we correlated brain graph dynamics with clinical variables.

Regression performance was measured using the coefficient of determination $R^{2}$ which is defined as

$$
R^{2} \equiv 1-\frac{\sum_{i=1}^{N}\left(y_{i}-f_{i}\right)}{\sum_{i=1}^{N}\left(y_{i}-\bar{y}\right)}
$$

where $\bar{y}=1 / n \sum_{i=1}^{N} y_{i}$. Thus the closer $R^{2}$ is to 1 , the better is the linear fit.

Table 1 summarizes this analysis. High scores were obtained for the proportion of intact and absolutely defected sectors (more or less independent 
Table 1 Regression scores $R^{2}$ for every pair of frequency band and clinical variable fitting $\operatorname{VAR}(1)$ (left) and $\operatorname{VAR}(2)$ (right)

\begin{tabular}{c|rrrrrr}
$p=1$ & $\delta$ & $\theta$ & $\alpha$ & $\beta$ & $\gamma$ & $\mu$ \\
\hline $\mathrm{w}$ & .198 & .727 & .715 & .276 & .207 & .370 \\
$\mathrm{~g}$ & .193 & .101 & .156 & .240 & .273 & .189 \\
$\mathrm{~b}$ & .226 & .605 & .692 & .328 & .269 & .400 \\
$\mathrm{w} C \mathrm{CMF}$ & .179 & .698 & .608 & .288 & .232 & .338 \\
$\mathrm{~g} C \mathrm{CMF}$ & .177 & .105 & .183 & .446 & .185 & .226 \\
$\mathrm{~b} C M F$ & .206 & .630 & .696 & .311 & .273 & .364
\end{tabular}

\begin{tabular}{c|rrrrrr}
$p=2$ & $\delta$ & $\theta$ & $\alpha$ & $\beta$ & $\gamma$ & $\mu$ \\
\hline $\mathrm{w}$ & .466 & .818 & .606 & .517 & .389 & .448 \\
$\mathrm{~g}$ & .276 & .328 & .608 & .376 & .391 & .318 \\
$\mathrm{~b}$ & .517 & .844 & .827 & .519 & .408 & .496 \\
$\mathrm{w}$ CMF & .470 & .822 & .590 & .551 & .389 & .434 \\
$\mathrm{~g}$ CMF & .319 & .331 & .584 & .403 & .318 & .341 \\
$\mathrm{~b}$ CMF & .516 & .850 & .819 & .526 & .406 & .471
\end{tabular}

from CMF). VAR models based on $\delta$ and $\alpha$ seem to produce suitable features. This regression analysis clearly shows that (1) we could actually find network features describing the dynamics of the weighted graphs and (2) these features correlate with the extend of the vision loss.

\section{Conclusion}

Recently neuroscientists started transforming neuroimaging data into brain networks. The idea behind this approach is to use graph theory and its algorithms to produce meaningful features that can help to understand brain recordings. Using any kind of time series similarity measure, the similarity of two data channels (nodes) at some point in time produces a new time series (edge incident to the nodes). The series of graphs is typically averaged to one network of which its edge weights are thresholded resulting in an unweighted network. We do not follow this "classical" approach as it omits the dynamics of the functional connectivity. Usually several graph measures are applied to differentiate between brain networks of distinct subjects or conditions.

Keeping the series of graphs thus creates a multivariate time series of these measures that need to be analyzed. Therefore in this paper we used model-based descriptions, i.e. VAR models. Regression was applied to find linear dependencies between VAR parameters and certain variables. An EEG application of visually impaired subjects showed that this approach is useful for the generation of features.

In the future, we want to work with specialized graph measures for brain networks that capture the high fluctuations and dynamics (see e.g. 11). Last not least, we hope to obtain more intuitive results using rule-based machine learning approaches to group or classify subjects based on learned models.

Acknowledgements. The authors thank Carolin Gall and her students from the Medical Faculty for collecting the EEG data. We give thanks to Hermann Hinrichs from the Medical Faculty for pointing out several hints to preprocess the EEG data. Finally, we thank the two anonymous reviewers for their very helpful comments to improve this paper. 


\section{References}

1. Bunke, H.: On a relation between graph edit distance and maximum common subgraph. Pattern Recognit. Lett. 18(8), 689-694 (1997)

2. Butts, C.T.: Revisiting the foundations of network analysis. Science 325(5939), 414-416 (2009)

3. Csárdi, G., Nepusz, T.: The igraph software package for complex network research. InterJournal Complex Systems 1695 (2006)

4. Daniel, P.M., Whitteridge, D.: The representation of the visual field on the cerebral cortex in monkeys. J. Physiol. 159(2), 203-221 (1961)

5. Delorme, A., Makeig, S.: EEGLAB: an open source toolbox for analysis of single-trial EEG dynamics including independent component analysis. J. Neurosci. Methods 134(1), 9-21 (2004)

6. Edwards, E.: Electrocortical activation and human brain mapping. PhD thesis, University of California, Berkeley, CA, USA (2007)

7. Held, P., Moewes, C., Braune, C., Kruse, R., Sabel, B.A.: Advanced Analysis of Dynamic Graphs in Social and Neural Networks. In: Borgelt, C., Gil, M.Á., Sousa, J.M.C., Verleysen, M. (eds.) Towards Advanced Data Analysis. STUDFUZZ, vol. 285, pp. 205-222. Springer, Heidelberg (2012)

8. Lütkepohl, H.: New Introduction to Multiple Time Series Analysis. In: Econometrics / Statistics. Springer, Heidelberg (2005)

9. Makeig, S., Bell, A.J., Jung, T., Sejnowski, T.J.: Independent component analysis of electroencephalographic data. In: Touretzky, D.S., Mozer, M.C., Hasselmo, M.E. (eds.) Advances in Neural Information Processing Systems, vol. 8, pp. 145-151. MIT Press, Cambridge (1996)

10. Montez, T., Linkenkaer-Hansen, K., van Dijk, B.W., Stam, C.J.: Synchronization likelihood with explicit time-frequency priors. Neuroimage 33(4), 11171125 (2006)

11. Pearl, J.: Causal inference in statistics: An overview. Stat. Surv. 3, 96-146 (2009)

12. Pedregosa, F., Varoquaux, G., Gramfort, et al.: Scikit-learn: Machine learning in python. JMLR 12, 2825-2830 (2011)

13. Rubinov, M., Sporns, O.: Complex network measures of brain connectivity: Uses and interpretations. Neuroimage 52(3), 1059-1069 (2010)

14. Sabel, B.A., Fedorov, A.B., Naue, N., Borrmann, A., Herrmann, C., Gall, C. Non-invasive alternating current stimulation improves vision in optic neuropathy. Restor. Neurol. Neurosci. 29(6), 493-505 (2011)

15. Sporns, O.: Networks of the Brain. MIT Press, Cambridge (2010)

16. Stam, C., Jones, B., Nolte, G., Breakspear, M., Scheltens, P.: Small-World networks and functional connectivity in alzheimer's disease. Cerebral Cortex 17(1), 92-99 (2007)

17. Stam, C.J., van Dijk, B.W.: Synchronization likelihood: an unbiased measure of generalized synchronization in multivariate data sets. J. Phys. D: Nonlinear Phenom. 163(3-4), 236-251 (2002)

18. Varela, F., Lachaux, J., Rodriguez, E., Martinerie, J.: The brainweb: phase synchronization and large-scale integration. Nat. Rev. Neurosci. 2(4), 229-239 (2001)

19. Watts, D.J., Strogatz, S.H.: Collective dynamics of 'small-world' networks. Nature 393(6684), 440-442 (1998)

20. Wendling, F., Ansari-Asl, K., Bartolomei, F., Senhadji, L.: From EEG signals to brain connectivity: A model-based evaluation of interdependence measures. J. Neurosci. Methods 183(1), 9-18 (2009)

21. Wüst, S., Kasten, E., Sabel, B.A.: Blindsight after optic nerve injury indicates functionality of spared fibers. J. Cogn. Neurosci. 14(2), 243-253 (2002) 